\title{
Preformed metal crowns for carious primary molars
}

\author{
A novel technique using preformed metal crowns for managing carious primary molars in general practice -
} A retrospective analysis N. P. T. Innes, D. R. Stirrups, D. J. P. Evans, N. Hall and M. Leggate

Br Dent J 2006; 200: ???-???

\section{Background}

There is a high level of untreated dental decay in primary teeth in Scotland. Despite evidence for the efficacy of preformed metal crowns (PMCs) for the restoration of primary molars, few are placed in general practice, possibly due to the interventive nature of the clinical procedure. There is, however, a novel way of placing PMCs involving no local anaesthesia, no caries removal and no preparation of the tooth: the Hall technique.

Aim

To investigate the survival of carious primary teeth treated with PMCs placed using a novel, simplified method - the Hall technique.

Setting

General dental practice, in Scotland.

Methods

A retrospective analysis of practice records from one general practitioner, from 1988 to 2001. The majority of the 978 PMCs fitted on 259 children, using the Hall technique, were placed when there was clinical evidence of approximal caries into dentine. The Kaplan-Meier approach was used to analyse survival times and the Mantel-Haenszel Log rank test for comparison between tooth types.

Results

For all tooth types, the probability of surviving three years without being extracted or the PMC being lost, was 73.4\% (95\% confidence interval $70.1 \%$ to $76.4 \%$ ), and for five years was $67.6 \%$ (95\% confidence interval $63.3 \%$ to $71.5 \%$ ). The probability of surviving without extraction alone for three years was $86.0 \%$ (95\% confidence interval $83.2 \%$ to $88.4 \%$ ), and for five years was $80.5 \%$ (95\% confidence interval $76.5 \%$ to $83.9 \%$ ). Conclusion

Hall technique restorations placed on primary molars with decay clinically into dentine, by a single operator in general dental practice, have a similar success rate to some other, more conventional, restorative techniques. The technique requires further evaluation through a prospective randomised control clinical trial before its use could be generally recommended.

\section{IN BRIEF}

- Highlights problems concerning the low level of restorative care provided for carious primary teeth in general practice.

- Presents audit data from a single GDP of a simplified method of placing preformed metal crowns, which does not involve LA or tooth preparation of any kind.

- Although the technique looks promising, the need for prospective randomised clinical trials before the technique is adopted, is stressed.

\section{COMMENT}

Treatment planning for patients with dental decay, be they children or adults, is more complicated than we often admit. Prevention must underpin all of our activity but there will be times when prevention, however assiduously pursued, will not be enough.

In the arena of dental care for children, there has been an unwarranted division between what is perceived as being 'general practice' care and what has been labelled 'specialist' care. This is a false distinction. Those with the technical skills to carry out good bridgework have all of the manual skills for paediatric restorative dentistry; it's just the 'paediatric' part that makes the difference, and that is often a matter of self-confidence!

Controlled trials are rare in this area, and often of relatively brief duration. However, those studies considering the use of preformed metal crowns (PMC), albeit limited by methodology, have all tended to point in the same direction. PMC are successful in maintaining teeth in health, even though those teeth have often been already heavily affected by caries.

But the problem of practitioner (non)-acceptance remains. The technique as classically taught requires the use of local anaesthesia and tooth preparation and from the lack of uptake for these or other reasons, it would seem that general practitioner colleagues in the main have set their faces against the use of PMC.

But what if we could find a technique with all of the stated benefits of PMC, but without the use of local anaesthesia or tooth preparation? Would this be enough to change colleagues' minds?

The paper by Innes etal. comes to this issue with forthright honesty. Here is the simplest technique possible for the use of PMC; and here are survival rates thoroughly compatible with work elsewhere in the literature; and yet the authors recognise all of the limitations of their retrospective audit and have eliminated case after case from their statistics in order to provide the most rigorous assessment of this technique. It would seem likely that if they had used other authors' criteria of 'success', their results would have been more impressive still.

I suspect that it is not too bold a claim to suggest that this paper may prove to be a landmark in the restorative dental care of children, as a part of total patient care. Dr Hall, for her novel approach to a recalcitrant problem, the new child patient with substantial caries and, with her, Drs Innes, Stirrups, Evans and Leggate, for their scrupulous approach to their work, are to be congratulated.

This is not a definitive answer, it is an initial, retrospective, audit, but we look forward with keen anticipation to the outcome of the prospective studies presently under way.

\section{P. Crawford, Paediatric Dentistry, University of Bristol}

doi: $10.1038 /$ sj.bdj.4813467 